Erratum

\title{
Erratum: Clay, L.; Paterson, M.; Bennett, P.; Perry, G.; Phillips, C. Early Recognition of Behaviour Problems in Shelter Dogs by Monitoring Them in Their Kennels after Admission to a Shelter. Animals 2019, 9, 875
}

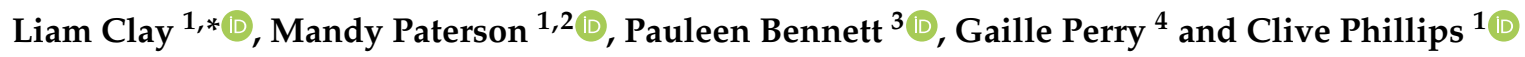 \\ 1 Centre for Animal Welfare and Ethics, University of Queensland, Gatton, Queensland 4343, Australia; \\ mpaterson@rspcaqld.org.au (M.P.); c.phillips@uq.edu.au (C.P.) \\ 2 Royal Society for the Prevention of Cruelty to Animals Queensland, Brisbane, Queensland 4076, Australia \\ 3 School of Psychology and Public Health, La Trobe University, Bendigo, Victoria 3552, Australia; \\ pauleen.bennett@latrobe.edu.au \\ 4 Delta Society, Summer Hill, New South Wales 2130, Australia; perrygaille@gmail.com \\ * Correspondence: liam.clay@uqconnect.edu.au; Tel.: +61-422-706-076
}

Received: 4 December 2019; Accepted: 10 December 2019; Published: 15 December 2019

The authors wish to make the following corrections to their paper [1]:

In Table 3, arousal was repeated as a row heading at the end-it should have read "friendliness" (see Table 3 below).

In Table 4, there were errors in the behaviours, their method of recording, the interactions and p-values. The new Table 4 is given below.

Scientific results were correctly stated in the text but incorrect in these two tables. The authors would like to apologize for any inconvenience caused. 
Table 3. The behaviours contributing to the emotional states of fear, anxiety, aggression, arousal, and friendliness.

\begin{tabular}{|c|c|c|c|c|c|c|c|c|c|c|c|c|c|c|c|}
\hline \multicolumn{16}{|c|}{ Behaviour Number } \\
\hline 1 & 2 & 3 & 4 & 5 & 6 & 7 & 8 & 9 & 10 & 11 & 12 & 13 & 14 & 15 & 16 \\
\hline \multicolumn{16}{|c|}{ Fear } \\
\hline Diverting & Ears Back & $\begin{array}{l}\text { Lip } \\
\text { Licking }\end{array}$ & $\begin{array}{l}\text { Lowered } \\
\text { Body }\end{array}$ & $\begin{array}{l}\text { Lowered } \\
\text { Head }\end{array}$ & Shiver & Stiff Tail & Tail Low & $\begin{array}{c}\text { Tail } \\
\text { Tucked }\end{array}$ & $\begin{array}{l}\text { Tense Body } \\
\text { Posture }\end{array}$ & $\begin{array}{l}\text { Weight } \\
\text { back }\end{array}$ & Yawn & & & & \\
\hline \multicolumn{16}{|c|}{ Anxiety } \\
\hline Fast tail & High tail & Jumping & Licking & $\begin{array}{l}\text { Lip } \\
\text { licking }\end{array}$ & Medium & Pacing & Panting & Stiff Tail & Tense body & $\begin{array}{l}\text { Weight } \\
\text { Back }\end{array}$ & $\begin{array}{l}\text { Weight } \\
\text { Forward }\end{array}$ & Whining & & & \\
\hline \multicolumn{16}{|c|}{ Aggression } \\
\hline Biting & $\begin{array}{c}\text { Ears } \\
\text { Forward }\end{array}$ & Growling & High Tail & $\begin{array}{l}\text { Lip } \\
\text { Licking }\end{array}$ & $\begin{array}{c}\text { Lowered } \\
\text { Head }\end{array}$ & $\begin{array}{l}\text { Medium } \\
\text { Tail }\end{array}$ & Snapping & Standing & Stiff tail & Still tail & Targeting & $\begin{array}{l}\text { Vertical } \\
\text { Lip Raise }\end{array}$ & & & \\
\hline \multicolumn{16}{|c|}{ Arousal } \\
\hline Barking & $\begin{array}{l}\text { Diverting } \\
\text { Gaze }\end{array}$ & Fast Tail & High Tail & $\begin{array}{l}\text { Jumping } \\
\text { Up }\end{array}$ & Jump Off & Licking & $\begin{array}{l}\text { Medium } \\
\text { Tail }\end{array}$ & Mouthing & Pacing & Panting & $\begin{array}{l}\text { Weight } \\
\text { Forward }\end{array}$ & Whining & & & \\
\hline \multicolumn{16}{|c|}{ Friendliness } \\
\hline Balanced & $\begin{array}{l}\text { Body } \\
\text { Curve }\end{array}$ & $\begin{array}{l}\text { Direct } \\
\text { eye }\end{array}$ & $\begin{array}{c}\text { Ears } \\
\text { Forward }\end{array}$ & $\begin{array}{l}\text { Ears } \\
\text { Open }\end{array}$ & Fast Tail & $\begin{array}{l}\text { Handler } \\
\text { Interaction }\end{array}$ & Jump & $\begin{array}{l}\text { Medium } \\
\text { Tail }\end{array}$ & Play & $\begin{array}{c}\text { Relaxed } \\
\text { body }\end{array}$ & slow & Sniff & Soft & $\begin{array}{c}\text { Tail } \\
\text { Loose }\end{array}$ & Walking \\
\hline
\end{tabular}

Table 4. Differences in kennel behaviour between dogs that were euthanased and adopted, either overall or on certain days.

\begin{tabular}{cccc}
\hline F/D & Behaviours & Interaction & $p$-Value \\
\hline D & Tense body & Day 1 & 0.001 \\
F & Balance/relaxed & Overall & 0.004 \\
F & Jumping in kennel & Overall & 0.03 \\
\hline \multicolumn{4}{c}{ D = Duration, F = Frequency. }
\end{tabular}




\section{Reference}

1. Clay, L.; Paterson, M.; Bennett, P.; Perry, G.; Phillips, C. Early Recognition of Behaviour Problems in Shelter Dogs by Monitoring them in their Kennels after Admission to a Shelter. Animals 2019, 9, 875. [CrossRef] [PubMed] 